\title{
QUIESCENT PROMINENCE EQUILIBRIA
}

\author{
TzIHONG CHIUEH ${ }^{1,2,3}$ AND LUPIN C. C. LIN $^{3}$ \\ Received 1998 April 14; accepted 1999 January 10
}

\begin{abstract}
With a new analytical method, we present a static model for quiescent solar prominences of inverse polarity. These prominences have a plasma $\beta$ slightly below unity and correspond to those located in the lower corona. Although this static model cannot address the helmet-like features at the top of the prominences, which are believed to be associated with the open-field topology and driven by outflows, the model may nevertheless capture several characteristic features of the solar prominences. These features include the sheetlike gas condensation hanging above an asymptotically weak magnetic cusp, the sheared magnetic arcades/loops, the coronal cavity, radio dark strips cospatial with the $\mathrm{H} \alpha$ filaments, bright radio ribbons located on both sides of the dense prominence sheet, and the increasing magnetic field strength with height along the prominence sheet. In our model, the densest gas is contained within a narrow field-free tube immediately above the prominence sheet. Thermally insulated by the surrounding strong fields, this narrow tube may be a natural site for housing the cool gas $\left(\geq 10^{4} \mathrm{~K}\right)$ siphoned from the lower solar atmosphere. We also find that there are hot spots $\left(\leq 10^{7} \mathrm{~K}\right)$ located on the sides of the prominence loop, which might be the source of thermal X-ray emission in the quiet Sun.
\end{abstract}

Subject heading: MHD - Sun: magnetic fields - Sun: prominences

\section{INTRODUCTION}

Quiescent prominences are long-lived, high-density gas sheets suspended in the lower corona by magnetic field tension (Martin 1989; Tandberg-Hanssen 1974, 1995; Tang 1987; Zirin 1988). The vertical gas sheets can extend horizontally up to a substantial fraction of the solar radius, i.e., a few times $10^{5} \mathrm{~km}$, and last for up to several months. The structure of quiescent prominences is robust, and it can remain intact even when occasional flares take place in the immediate neighborhood. Quiescent prominences may also end by suddenly undergoing spectacular eruptions, lifting the dense gases high (about 1 solar radius) into the solar corona and eventually ejecting these plasmoids into interplanetary space (Low 1996; Gibson \& Low 1998). Although such intermittent mass ejection, the so-called "coronal mass ejections" (as opposed to the mass loss from the quasisteady solar wind) account for only a negligible fraction of the total mass loss from the Sun, coronal mass ejection is now thought to be the primary mechanism for removing the magnetic helicity from the interior of the Sun (Low 1996; Chiueh 1998).

On the solar disk, the quiescent prominences appear as dark filaments, because of the enhanced absorption of photospheric light, while above the solar limb they appear as bright filaments, indicating a high-density gas associated with the filaments. In addition, high-resolution $\mathrm{H} \alpha$ observations of the solar disk show that the prominence consists of many well-organized, short fibril structures, oriented in directions branching away from the general direction of the averaged prominence filament with acute angles (Leroy

\footnotetext{
${ }^{1}$ Physics Department, National Taiwan University, Taipei, Taiwan; chiuehth@phys.ntu.edu.tw.

${ }^{2}$ Institute of Astronomy and Astrophysics, Academia Sinica, Taipei, Taiwan.

${ }^{3}$ Institute of Astronomy, National Central University, Chung-Li, Taiwan.
}

1989). These fine $\mathrm{H} \alpha$ fibrils trace the magnetic fields, which are an important agent for the formation of the prominences. The magnetic field associated with the prominence provides the support needed to suspend the dense cool gas up in the solar corona. The cool gas condensed in the prominence may result from thermal instabilities of the high-temperature corona plasma, which is radiatively cooled by both line emissions and Bremsstrahlung processes (Field 1965; Hildner 1974). The material within the prominence could be supplied by siphoning the gas from the lower atmosphere along some magnetic channels.

However, in the presence of solar gravity, such a prominence configuration tends to be unstable to the RayleighTaylor instability. Since the quiescent prominence is observed to be long lived even when it is subject to large disturbances such as flares, these magnetic structures must contain strong stabilization components against the interchange of field lines associated with the Rayleigh-Taylor instability. One effective arrangement of field configuration that resists the interchange of field lines includes magnetic shear (Cattaneo, Chiueh, \& Hughes 1990a, 1990b). In spite of this general principle for the stabilization of the interchange instability, the magnetic shear must be distributed in an appropriate way; otherwise, the magnetic system can be subject to another type of instability. This is because the magnetic shear is created by electric currents, and their presence introduces additional free energy into the system. For example, kink instabilities are the typical consequences of excessive current density in the magnetic systems (Chiueh \& Zweibel 1987, 1989). Thus, the longevity of prominences must require some delicate balance that can suppress the release of both magnetic and gravitational free energy from the systems.

As a first step toward a good understanding of solar prominences, our strategy is to systematically construct a series of prominence equilibria, whose stabilities and physical properties will be examined in detail in the next stages. In particular, through this first step it should be possible to 
investigate the essential mechanisms that might give rise to the prominence eruption, which yields the spectacular coronal mass ejection events.

In this work, we focus on the construction of prominence equilibria. A new mathematical method is introduced for the construction of a particular class of two-dimensional prominence equilibria that possess three components of the magnetic field, with a range of magnetic shear strengths. The prominence sheets so constructed are found to be in inverse polarity, configurations in which the gas condenses at the bottom of closed magnetic loops suspended in the lower corona. This new mathematical technique is analyti$\mathrm{cal}$, and it turns out that the resulting equation is exceedingly simple. Nonetheless, it does contain sufficient freedom to allow us to construct various equilibria by "cut-andpaste" operations whenever we needs to describe field configurations with sufficient complexity.

Cut-and-paste is possible for our equilibrium construction because we demand that the gas along each field line is always in pressure balance with a corresponding fiducial atmosphere, and that different field lines match the fiducial atmospheres of different pressures. With this mathematical technique, the solution can always be truncated at any flux surface, and it can always be in pressure balance with the background atmosphere, which is also one of the family of fiducial atmospheres. In addition, guided by observations, whenever an additional magnetic structure of a different shape is required, one can simply insert a new magnetic structure into the existing system, together with a volume of magnetized background gas in between the geometrically different magnetic structures. The inserted magnetized gas can serve as a pressure blanket between these magnetic structures, and by construction this gas blanket can be easily adjusted to be in pressure balance with the existing magnetic structures. From the physical point of view, the matching of magnetic structures through the introduction of a magnetized background gas is reasonable, since the different magnetic structures can represent the magnetic flux either emerging to the solar surface at different epochs or having undergone drastic changes in the field-line topology; therefore, the magnetic flux can be enclosed by, or enclosing, a volume of background plasma already present in the solar atmosphere. From the technical point of view, this cut-and-paste procedure allows one to construct a variety of field shapes according to the requirements from the observations. The advantage of this freedom is unprecedented, as compared to the conventional method that directly solves the nonlinear Grad-Shafranov equation; solution construction through the Grad-Shafranov equation usually has little control over the shapes of the magnetic structures obtained. The present method also has a great advantage over the conventional self-similar analyses, which allow only for one type of self-similar solution over the entire domain, as opposed to the coexistence of several types of self-similar solutions presented here (Blandford \& Payne 1982; Li, Chiueh, \& Begelman 1992). Unfortunately, the present method is limited to the investigation of static plasmas, and therefore cannot faithfully describe the physics outside the magnetic loops and arches in the open atmosphere, which is dominated by coronal streamers. To obtain the pressure of the open atmosphere required to confine prominence loops and arches, we adopt a static atmosphere with a simple field configuration. Hence, our results for the open atmosphere should be interpreted with caution.
This paper is organized as follows. Section 2 contains the mathematical formulation of this analytical approach. Section 3 gives examples of the detailed solutions. Discussions and conclusions are given in $\S 4$.

\section{MATHEMATICAL FORMULATION OF THE QUASI-SELF-SIMILAR MAGNETIC STRUCTURES}

As in any physical system, a scale-invariant, self-similar nonlinear solution can be constructed whenever the relevant dynamics has no characteristic length scale. In the context of the solar atmosphere, the magnetic pressure scale height in the corona is about $10^{5} \mathrm{~km}$, and the typical prominence transition thickness is about a few $\times 10^{2} \mathrm{~km}$; any nonlinear object with a length scale in between these two disparate scales is likely to assume a scale-invariant form.

A self-similar system usually possesses a dimensional scaling variable, the availability of which is often determined by whether the physical system contains no more than two independent dimensional constants (Sedov 1959). However, in many situations self-similarity is a bad assumption. An equilibrium configuration can be established through different stages of evolution, and the timescales, initial conditions, and boundary conditions involved may introduce some characteristic length scales even though the original dynamical equations contain no characteristic length. The length scale introduced in such an indirect way can often appear in the course of the analysis as additional dimensional constants. For example, the explosive energy in Sedov's self-similar solutions for blast waves is a dimensional constant. With the presence of these additional constants, the scale-invariant symmetry in a seemingly scale-invariant system may be broken.

The quasi-self-similar solutions introduced in the present work are devised to circumvent the aforementioned problem. Specifically, we hope to paste different families of self-similar solutions together to construct a global solution, in which each family of the self-similar solution corresponds to a particular physical condition under which the local system forms. However, different families of selfsimilar solutions can have their own characteristic shapes. Hence, matching different families of self-similar solutions together is by no means an obvious task. For example, it is impossible to match a family of self-similar triangles enclosed by a family of self-similar rectangles. Although one side of the outermost triangle can be pasted onto one side of the innermost rectangle, other parts of the triangle usually cannot be matched onto the rectangle, thus leaving a "vacuum" in between the two families of solutions.

The resolution to the problem of matching different families of solutions has already been hinted at in the above example. One needs only to fill in the vacuum with a third, "easy" solution that can match onto these different selfsimilar solutions. In the present context, the third solution consists of a volume of background gas permeated by horizontal fields. The background fields tend to be geometrically simple, and we assume that they contain straight field lines; this simplification allows us to treat the background fields as scalar fields that provide only pressure force in the force balance. Such a background magnetized gas can be made to be in pressure balance with the different classes of self-similar magnetic structures with relative ease. Since the background gas is gravitationally stratified, the pressures on different matching boundaries can be rather different. In order to meet our goal, the self-similar magnetic structures 
must possess a special property that permits such pressure matching. In this section we present an analysis describing how such a useful property of magnetic structures can be implemented.

Consider a three-component magnetic field $\boldsymbol{B}$, with its strength depending on $x$ and $z$, where $z$ is the direction against the gravity. Let $\boldsymbol{B}=B_{h} \hat{y}+\boldsymbol{B}_{p}$, where $\boldsymbol{B}_{p}$ is the component that is perpendicular to the invariant direction $y$ and satisfies $\boldsymbol{B}_{p}=\hat{y} \times \nabla \psi, \psi$ being the flux function. This representation of $\boldsymbol{B}_{p}$ warrants $\boldsymbol{\nabla} \cdot \boldsymbol{B}_{p}=0$. (We let the background horizontal field be oriented in the $y$ direction.) For an equilibrium configuration, the balanced force in the $y$ direction reads: $\hat{y} \times \nabla \psi \cdot \nabla B_{h}=0$, and it follows that $B_{h}$ must be a function of $\psi$, i.e., $B_{h}=B_{h}(\psi)$. So far, this has been a standard approach to tackling two-dimensional equilibria.

We now assume the following self-similar scaling relations: $z=\psi^{\alpha} \eta, x=\psi^{\alpha} \xi(\eta), B_{h}=\psi^{1-\alpha} b, T=\psi^{\alpha} t(\eta)$, and $\rho=\psi^{2-3 \alpha} \tau(\eta)$, where $T$ and $\rho$ are the temperature and plasma density, respectively, and $b$ is a constant. The scaled quantities, such as $\xi, t$, and $\tau$, are expressed in terms of the self-similar variable $\eta$, which measures the height of the reference field line, assuming $\psi=1$. Let

$$
\frac{\xi(\eta)}{\eta}=f(\eta)
$$

or, equivalently,

$$
\eta=f^{-1}\left(\frac{\xi(\eta)}{\eta}\right),
$$

where $f^{-1}$ is the inverse of the yet-to-be-determined function $f$. The geometric meaning of $\xi / \eta$ is simply the arctangent of the angle from the $z$-axis, and in equation (1) we attempt to express the angle in terms of the height $\eta$. To trace the field-line trajectory we then use the relations

$$
B_{z}=\frac{\partial \psi}{\partial x}=\frac{\partial}{\partial x}\left(\frac{z}{\eta}\right)^{1 / \alpha}=\frac{\psi^{1-\alpha}}{\alpha} \frac{\partial}{\partial x}\left(\frac{z}{f^{-1}}\right) .
$$

With the chain rules,

$$
\begin{aligned}
\frac{\partial}{\partial x}\left(\frac{z}{f^{-1}}\right) & =-\frac{z}{\left(f^{-1}\right)^{2}}\left[\frac{\partial}{\partial x}\left(\frac{x}{z}\right)\right] \frac{d f^{-1}}{d(x / z)} \\
& =-\frac{1}{\eta^{2}} \frac{d f^{-1}}{d(x / z)} \\
& =-\frac{1}{\eta^{2}} \frac{1}{[d(\xi / \eta)] / d \eta} \\
& =\frac{1}{\xi-\eta \dot{\xi}},
\end{aligned}
$$

we now obtain

$$
B_{z}=\frac{\psi^{1-\alpha}}{\alpha(\xi-\eta \dot{\xi})},
$$

where $\dot{\xi} \equiv d \xi / d \eta$. Likewise, one can follow similar steps to obtain

$$
B_{x}=\frac{\psi^{1-\alpha \dot{\xi}}}{\alpha(\xi-\eta \dot{\xi})} .
$$

This yields $B_{x} / B_{z}=\dot{\xi}$, which indeed traces the trajectory of the reference field line. Equations (4) and (5) represent the magnetic field in terms of a nonorthogonal coordinate, $(\psi$, $\eta)$.

This representation has been discussed previously and applied to the study of self-similar magnetohydrodynamic (MHD) jets emanating from accretion disks for both nonrelativistic (Blandford \& Payne 1982) and relativistic (Li et al. 1992) cases. Unlike these previous works, which consider the entirely scale-free, self-similar solutions, the present work employs a new method, which we call the quasi-selfsimilar method. This method permits the coexistence of different classes of truncated self-similar solutions in the same space. It is possible because we give up an a priori assumption of the equation of state, since the energy transport in the solar atmosphere is a problem that is yet to be settled; this degree of freedom is replaced by the requirement that different classes of self-similar structures must be pressurematched onto the same background atmosphere. Without this essential step, solution matching among different classes of self-similar solutions is generally impossible, and this is why the conventional self-similar solution admits only one type of self-similarity over the entire solution domain.

We now consider the force balance along the poloidal field line. Let $\boldsymbol{B}_{p} \cdot \nabla \equiv B_{p} d / d s$, where $s$ is the distance along the poloidal field line and $B_{p} \equiv \psi^{\alpha-1}\left(1+\dot{\xi}^{2}\right)^{1 / 2} / \alpha(\xi-\eta \dot{\xi})$. The forces along the field line satisfy

$$
\frac{d}{d s}(\rho T)+\frac{g \rho \hat{z} \cdot B_{p}}{B_{p}}=0,
$$

which can be formally integrated to become

$$
\tau(\eta) t(\eta)=p_{0} e^{-g \int d \eta / t(\eta)} \equiv p_{0} F(\eta),
$$

where $\hat{z}$ is the unit vector opposite to gravity and $p_{0}$ is an integration constant. Thus, $\tau$ and $t$ are not independent and are related by equation (7).

Next, we consider the force balance across the flux surface:

$$
\begin{aligned}
& -\hat{\psi} \cdot \nabla\left[\left(B_{p}^{2}+B_{h}^{2}\right) / 2+p_{0} F(\eta) \psi^{2-2 \alpha}\right] ; \\
& +\hat{\psi} \cdot\left(\boldsymbol{B}_{p} \cdot \nabla \boldsymbol{B}_{p}\right)-g \rho \hat{\psi} \cdot \hat{z}=0,
\end{aligned}
$$

where $\hat{\psi}$ is the unit vector perpendicular to the magnetic flux pointing toward the direction of increasing $\psi$. We need four preparatory steps before equation (8) can be evaluated. First, $\hat{\psi} \cdot \hat{z}=\nabla \psi \cdot \hat{z} / B_{p}=\dot{\xi}\left(1+\dot{\xi}^{2}\right)^{-1 / 2}$, from equations (4) and (5). Second, $\hat{\psi} \cdot\left(\boldsymbol{B}_{p} \cdot \nabla \boldsymbol{B}_{p}\right)=\kappa B_{p}^{2}$, where $\kappa$ is the local curvature of the poloidal field line, given by

$$
\kappa=\ddot{\xi}\left(1+\dot{\xi}^{2}\right)^{-3 / 2} \psi^{-\alpha},
$$

which assumes a positive value when the field lines curve away from the origin $(\xi=\eta=0)$. Third, $\hat{\psi} \cdot \nabla=B_{p}(\partial / \partial \psi)$ $+\psi \cdot \nabla \eta(\partial / \partial \eta)$, and

$$
\hat{\psi} \cdot \nabla \eta=\psi^{-\alpha}\left[\frac{\eta+\xi \dot{\xi}}{\left(1+\dot{\xi}^{2}\right)^{1 / 2}(\xi-\eta \dot{\xi})}\right] .
$$

The expressions for equations (9) and (10) can be straightforwardly derived by chain rules similar to those used to obtain equations (4) and (5).

The final step before evaluating equation (8) involves a new method that permits solution matching in the presence of different families of self-similar solutions. We demand that the total pressure always remain the same as the total 
pressure of a fiducial magnetized gas:

$$
\frac{B_{p}^{2}+B_{h}^{2}}{2}+p_{0} F(\eta) \psi^{2-2 \alpha}=p_{e 0} e^{-\eta / L_{0}} \psi^{2-2 \alpha},
$$

where the right-hand side is the total pressure of the fiducial gas, $L_{0}$ is its pressure scale height, and $p_{e 0}$ is the fiducial pressure evaluated at $\eta=0$ and $\psi=1$. The magnetic field of the fiducial gas is assumed to be along the $\hat{y}$ direction and hence can be treated as a scalar field. In fact, we do not need to assume that the total pressure of the fiducial magnetized gas obeys an exponential form, as expressed on the righthand side of equation (11). However, the exponential form conventionally represents a stratified isothermal atmosphere, and so we follow this convention, with the understanding that $L_{0}$ should be regarded as the local scale height. (In fact, we could have assumed the fiducial pressure to be any arbitrary function of height without introducing any complication to the following analysis.)

Note that with the addition of the magnetic pressure, the length scale $L_{0}$ is actually larger than that of a field-free gas. For example, take the fiducial gas to be the background gas in the corona. At a corona temperature of about a few $\times 10^{6} \mathrm{~K}$, the pressure scale height of an unmagnetized gas is about several $\times 10^{4} \mathrm{~km}$, but the scale height of the total pressure $L_{0}$ can be as large as $10^{5} \mathrm{~km}$.

Equation (11) also allows one to truncate the self-similar solution anywhere one wishes and match it to the fiducial gas solution. With equation (11) in place, the first term of equation (8) may attain a simple expression at the cost of the third term becoming complicated. Note from equation (7) that $d F(\eta) / d \eta=-g F(\eta) / t(\eta)$, and hence the quantity $g \tau$ in the last term of equation (8) becomes

$$
g \tau=-p_{0} \frac{d F}{d \eta}=\frac{d}{d \eta}\left[\frac{1+\dot{\xi}^{2}}{2 \alpha^{2}(\xi-\eta \dot{\xi})^{2}}\right]+\frac{p_{e 0}}{L_{0}} e^{-\eta / L_{0}}
$$

by virtue of equations (4), (5), and (11).

After all these building blocks have been implemented, the final equation can be straightforwardly obtained from equation (8), and it takes an exceedingly simple form:

$$
\ddot{\xi}+c e^{-\eta}\left(c_{1}-\eta\right)\left[\frac{(\xi-\eta \dot{\xi})^{2}}{\xi}\right]=0,
$$

where $c \equiv \alpha^{2} p_{e 0} L_{0}^{2} / \psi_{0}^{2}, c_{1} \equiv 2(1-1 / \alpha)$, and $\eta$ and $\xi$ have been normalized to the pressure scale height $L_{0}$ of the fiducial magnetized gas. We emphasize that although equation (13) is derived with all physical quantities evaluated at the reference flux surface $\psi=\psi_{0}$, this equation remains valid for all flux surfaces as well, because of self-similarity. As will be shown later, all valid solutions always have a large $c$. To understand what this means, we can rearrange $c$ as $\alpha^{2}\left[p_{e 0} /\left(\psi_{0}^{2} / L_{b 0}^{2}\right)\right]\left[L_{0} / L_{b 0}\right]^{2}$, where $L_{b 0}$ is the characteristic length of the poloidal magnetic structures on the reference field line $\psi=\psi_{0}$. The first square bracket is of order unity because of the pressure balance, and therefore the magnitude of $c$ is largely determined by the squared ratio of the background pressure scale height $L_{0}$ to the typical length $L_{b 0}$ of the poloidal magnetic structures. The large value of $c$ simply implies small magnetic structures as compared to the large-scale background fields in the corona, i.e., $L_{0} \gg$ $L_{b 0}$. That is, we are limited to solutions for the low-lying prominences of a height of about $2 \times 10^{4} \mathrm{~km}$.

The resulting equation, equation (13), traces the trajectory of the reference field line and has a singularity at $\xi=0$.
By varying $c$ and $c_{1}$, we can obtain field lines of different shapes. Note that equation (13) is invariant to rescaling of $\xi$. That is, the solution of a thin magnetic loop can always be transformed to that of a fat loop by rescaling $\xi$. However, there is a caveat regarding such a scale transformation, since other considerations must be taken into account in order to prevent false solutions. In fact, solutions obtained by such a scale transformation have very different physical properties from the original ones, and some may even become unphysical in giving negative densities, as will be discussed in $\S 3$.

\section{PROMINENCE SOLUTIONS}

Two different types of solutions can be constructed from equation (13). When $\eta<c_{1}$, the solution $\xi(\eta)$ is of convex shape with a negative curvature, as viewed from the origin $(\xi=\eta=0)$; when $\eta>c_{1}$, on the other hand, the solution $\xi(\eta)$ has a positive curvature. The former can give a closedfield configuration and the latter an open-field configuration. It turns out that the latter always yields negative background pressure well above the photosphere. This is in part because the open field line region can no longer remain in static equilibrium, and our assumption of a static equilibrium only leads to false solutions. Hence, from now on we will discard these positive-curvature solutions in our pursuit of static equilibria.

Only within a range of parameters can the negativecurvature solutions yield positive-density solutions. The field line, starting its trajectory at the top (on the $\eta$ axis with $\eta>0$ ), may smoothly reach the negative $\eta$ axis at the bottom to form a closed magnetic loop. However, at the very bottom of the loop, $\eta_{b}$, the field line is always not of a parabolic shape, but of a shape that can be described by $\eta-\eta_{b} \propto \xi^{1+k}$, where $0<k<1$. Thus, a magnetic cusp exists at the loop bottom, where the dense prominence filament is expected to hang. For the prominence loops to be entirely visible above the photosphere, they must be located at a level above the photosphere, i.e., $z=\eta \psi^{\alpha} \geq z_{\mathrm{ph}}=$ $\eta_{\mathrm{ph}} \psi^{\alpha}$. On the other hand, for those loops that have $\psi$ values violating the above condition, the loop bottoms are located beneath the photosphere, and these loops are then viewed as the magnetic arcades. The above qualitative descriptions will be elaborated further in this section.

In principle, the closed-loop solution and the arcade solution can coexist, represented by two different families of self-similar solutions. One can insert layers of magnetized gas in between the loop and the arcade to fill up the space. In this work, we present two types of solutions, the first being simple magnetic loops immersed in a common magnetized background gas, the other being interacting loop/ arcade systems that consist of different families of self-similar solutions, also immersed in a magnetized background gas. When the observed prominences exhibit configurations more complicated than the example given in this work, one can always increase the structural complexity by inserting more arcades or loops of different families of solutions into the simpler solutions.

\subsection{Simple Magnetic Loop Solution}

We now present the solution of a single self-similar loop. Equation (13) can be integrated starting from the top of the reference magnetic loop, which is located at $\xi=0$ and $\eta=$ $\eta_{t}$. Since $\xi=0$ is a singularity of equation (3), we can expand the solution around the loop top. When we require 
that the field-line shape be parabolic at the top, we get the near-axis solution: $\xi=a\left(\eta_{t}-\eta\right)^{1 / 2}$, where $a$ is the amplitude of the solution. Because of the invariance in rescaling $\xi$ for equation (13), the quantity $a$ can, at this point, be regarded as a free parameter. (However, the quantity $a$ should eventually be constrained within a certain range, as shown below.) Inserting the near-axis solution into equation (13), we obtain a condition for $\eta_{t}$ :

$$
\eta_{t}^{3}-c_{1} \eta_{t}^{2}+\frac{e^{\eta_{t}}}{c}=0
$$

For positive $c$ and $c_{1}$, there are only two positive roots to equation (14), $\eta_{t+}$ and $\eta_{t-}$, where $\eta_{t+}>\eta_{t-}$. Hence, there are two different initial heights from which integration of equation (13) can be started, and they yield two different field line trajectories.

For a closed loop, the integration will encounter another point $\xi=0$ at the loop bottom. To investigate how the field lines look at the bottom, $\eta_{b}$, we now solve for a more general near-axis solution. Equation (13) near $\xi=0$ becomes

$$
\frac{\xi \ddot{\xi}}{(\dot{\xi})^{2}}=c e^{-\eta_{b}}\left(\eta_{b}-c_{1}\right) \eta_{b}^{2} \equiv-k
$$

where $\eta_{b}(<0)$ is the level of the loop bottom. A straightforward integration of equation (15) shows that

$$
\xi \sim\left(\eta-\eta_{b}\right)^{1 /(1+k)} .
$$

When $k=1$, we recover the parabolic shape, and the condition for $k=1$ has been given in equation (14). However, for the prominence gas to be highly condensed at the bottom of the loop, it is desirable that the loop bottom should have a $V$-shaped cusp field whose tension force balances the weight of the dense gases. Thus, we look for solutions in which

$$
0<k<1
$$

at the loop bottom. Note that since $d \eta / d \xi \sim \xi^{k}$, the slope of the field line is not finite but zero for $0<k<1$. Hence, such a magnetic cusp is actually an asymptotically weak cusp, which has a local curvature that diverges as $\kappa \sim \xi^{k-1}$.

Integration of equation (13) employs a second-order Runge-Kutta scheme to avoid errors near the singularity at $\eta_{b}$. The integration shows that for all positive $c$ 's, the solutions at the loop bottom $\eta_{b}$ always satisfy equation (17), whether we begin the integration from $\eta_{t+}$ or from $\eta_{t-}$, indicating that the cusp field at the loop bottom should be a generic feature of the self-similar equilibria. We further find that the solutions starting from $\eta_{t+}$ always have higher loop bottoms than the solutions starting from $\eta_{t-}$. As a result of the dependence of $k$ on $\eta_{b}$ (see eq. [15]), it follows that the former has a smaller value of $k$, i.e., a sharper cusp, than the latter. These two seemingly plausible trajectories starting from different heights turn out to yield not always physical solutions. In particular, the solutions starting from $\eta_{t+}$ always fail to yield positive density throughout the entire loop, and hence should be discarded. A positive value of the gas density thus imposes a strong constraint in limiting the valid ranges of $c, c_{1}$, and the amplitude $a$ for the trajectories starting from $\eta_{t-}$.

The gas density $\tau$ at the reference field line (for which $\psi=\psi_{0}=1$ ) has been given in equation (12). Together with equation (13), it is simplified to become:

$$
\tau=\frac{c}{g}\left(1-\frac{c_{1}}{2}\right)^{2} e^{-\eta}\left[1-\left(c_{1}-\eta\right) \frac{\eta+\xi \dot{\xi}}{\xi(\xi-\eta \dot{\xi})}\right] .
$$

Having determined $\xi(\eta)$ from the integration of equation (13), we can, at the same time, determine the density $\tau$ from equation (18). The region of gas density that is most likely to become negative is located where the magnetic loop turns halfway around, with the field-line direction pointing in the vertical direction and $\dot{\xi} \rightarrow 0$; meanwhile, the value of $\eta$ is always positive at this location. This observation suggests that the strategy for seeking the parameter regime of the physical solutions should be simply to search for the regime boundaries of $c, c_{1}$, and $a$ such that the gas density at $\dot{\xi}=0$ is barely positive.

Among these parameters, the most sensitive one is $c_{1}$, which, according to our numerical results, must lie in the range

$$
0<c_{1}<0.765
$$

in order to yield positive-density solutions. When $c_{1}$ assumes values near the lower limits, the quantity $c$ must approach infinity, and when $c_{1}$ is near the upper limit, $c$ assumes a value of about 332. Within this parameter domain, there always exists a range of amplitude $a$ such that the density can be made positive and definite. However, near the upper $c_{1}$ limit, the range of $a$ shrinks to a point at 1.12. The range of physical solutions in the $\left(c, c_{1}\right)$ phase space is plotted in Figure 1; it lies to the right of the boundary curve marked by circles.

Since it is expected that the gas should condense at the magnetic cusp, we now give an analysis of the gas density near the loop bottom, where a magnetic cusp exists. Substituting the solution given in equation (16) into equation (18), we find that

$$
\tau\left(\eta \rightarrow \eta_{b}\right) \sim\left(\eta-\eta_{b}\right)^{1-[2 /(1+k)]} \sim \xi^{k-1},
$$

where the second relation is from equation (16). Since $0<k<1$ (see eq. [17]), we indeed find that $\tau \rightarrow \infty$ as $\eta \rightarrow \eta_{b}$. Although the gas density diverges at the loop bottom, it still contains a negligible mass near this location, since $\int \tau d \xi \sim\left(\eta-\eta_{b}\right)^{2 k /(1+k)} \rightarrow 0$. This result is consistent with the fact that the magnetic cusp is an asymptotically

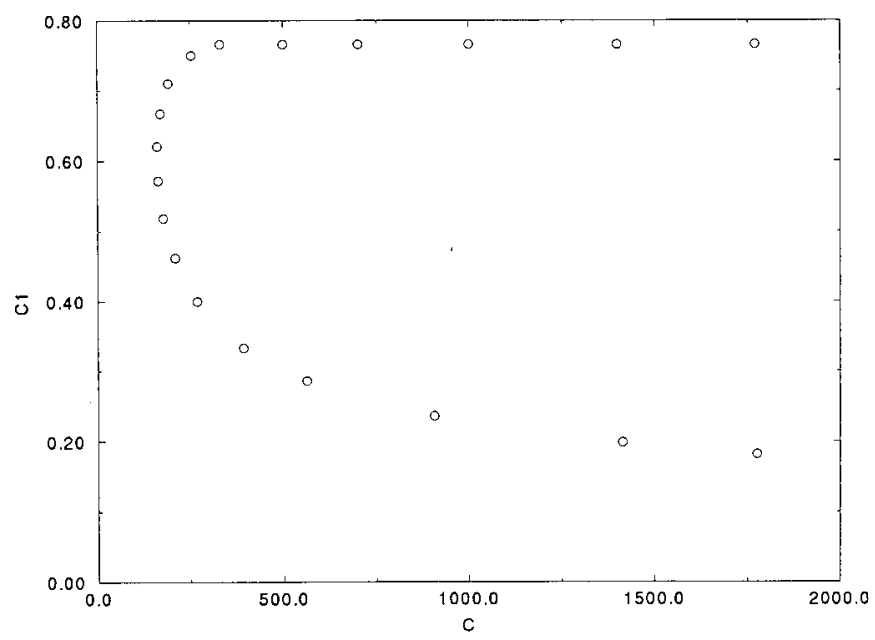

FIG. 1.- Regime of positive-density solutions in the $\left(c, c_{1}\right)$ phase space, which lies to the right of the boundary curve marked by the circles. 
weak cusp, for which the local tension force also diverges as $\xi^{k-1}$, in agreement with the local gravity, $g \tau$.

To end this section, we also examine the positivity of the gas pressure, which is expressed as

$$
p_{0} F(\eta)=p_{e 0} e^{-\eta}-\frac{b^{2}}{2}-\frac{1+\dot{\xi}^{2}}{2 \alpha^{2}(\xi-\eta \dot{\xi})^{2}}
$$

on the reference field line. Although the pressure given in equation (21) is derived in quite a different way from the density, it turns out from our numerical results that in the parameter regime where the gas density is positive, the gas pressure can always be positive for a sufficiently small magnetic pressure, $b^{2} / 2$, of the $y$-component field. The gas pressure is rather uniform along the poloidal field line, and hence there always exist hot spots at the density minima on the two sides of a magnetic loop. This feature deserves more attention, and will be discussed further in $\S 4$.

\subsection{Multiple Simple Prominence Solutions}

The effective pressure $\left[p_{0} F(\eta)+b^{2} / 2\right]$ and density along the reference field lines are plotted in Figure 2 as functions of $\eta$; the solid lines represent the large loop and the dashed line the small loop of Figure 3. In Figures $3 a$ and $3 b$, we depict the field lines and gas density of two nearby prominences. In between the two prominences, the space is filled by an isothermally stratified magnetized gas. The prominence on the left is constructed with the parameters $c_{1}=$ 0.33 and $c=450$, and the one on the right with $c_{1}=0.67$ and $c=1330$. The amplitude $a$ for each is chosen in such a

(a)

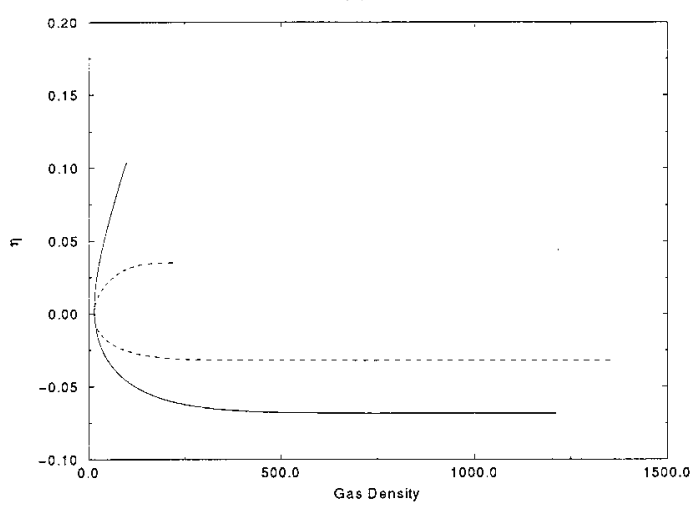

(b)

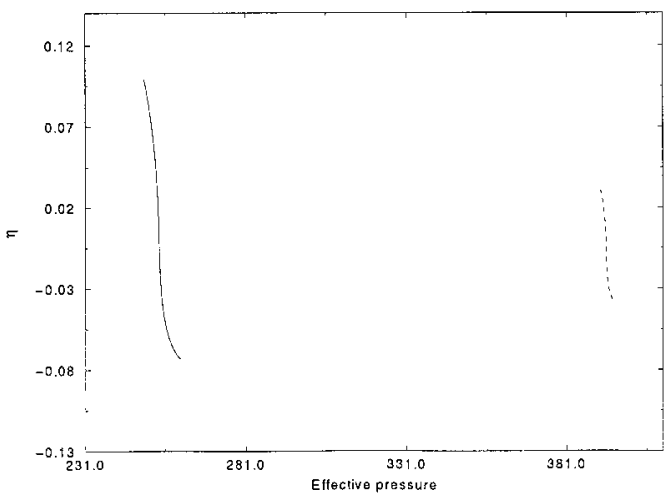

FIG. 2.-(a) Density profiles and (b) effective pressure profiles along the reference field lines for the large (solid lines) and small (dashed line) loops of Fig. 3. (a)

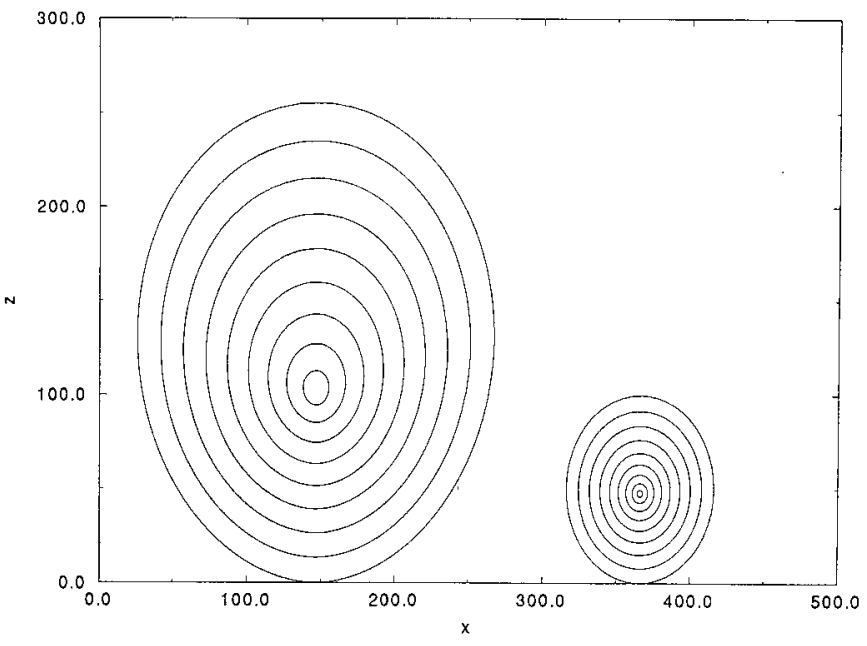

(b) $\log ($ Density)

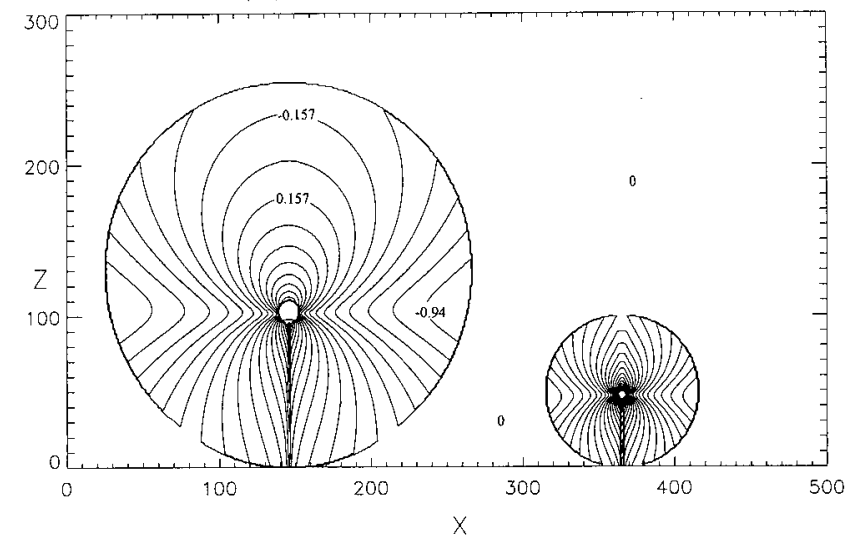

(c) $\log ($ Temperature)

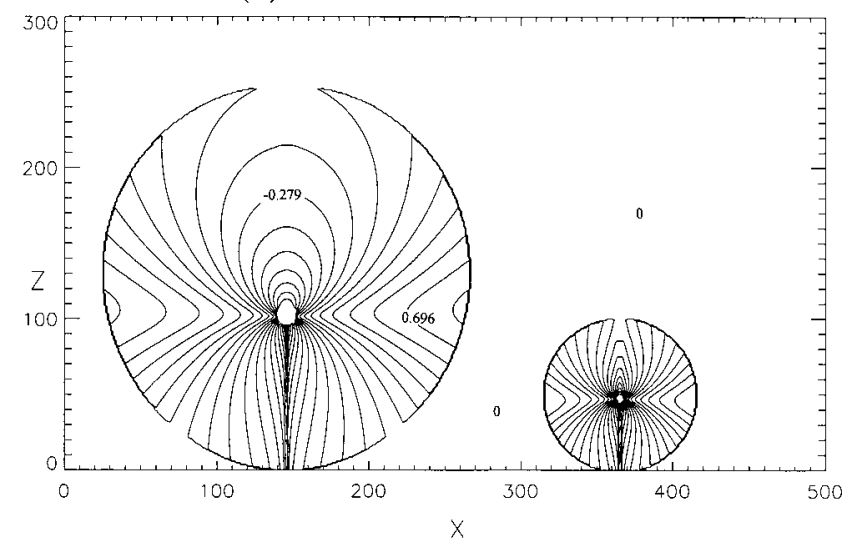

FIG. 3--Equilibrium configuration of two adjacent, noninteracting prominences. Shown are contour plots of $(a)$ the poloidal magnetic flux, $(b)$ $\log _{10}(\rho)$, and $(c)$ and $\log _{10}(T)$, respectively. The adjacent $\log _{10}(\rho)$ contours differ by a constant value of 0.157 , and those for $\log _{10}(T)$ differ by 0.14 .

way that it minimizes the ratio of the density at the loop top to the minimum density on the two sides of the magnetic loop. The values of $a$ are 1.265 and 0.834 for the large and small loops, respectively. Apart from their slight differences in shape, both prominence loops share similar physical properties, in that they are hot on the sides and dense at the 
bottoms of the closed loops. Note that most areas enclosing the dense sheets in the loop have low densities; we can identify them to be the coronal cavities. We choose the quantity $b$ such that the temperatures at the loop tops are the same as the local ambient plasma; the temperature profile of this composite system is shown in Figure $3 c$. The ratio $\left|b_{p}(\eta) / b\right|$ for the two loops is shown in Figure 4, where the solid line represents the large loop and dashed line the small loop; this figure shows that the magnetic fields in the loops are sheared.

Each of the two magnetic loops is constructed according to the procedure described in $\S 3.1$. In constructing the two loops, the centers of self-similarity, $(\eta=0, \xi=0)$, have been placed at different heights, such that the bottoms of both loops are located at levels with the same background pressure, i.e., at the photosphere. Such a condition can be expressed as

$$
\left.\left(\frac{c}{\alpha^{2}}\right)\right|_{1} \psi_{1}^{2-2 \alpha_{1}} e^{\eta_{b 1}}=\left.\left(\frac{c}{\alpha^{2}}\right)\right|_{2} \psi_{2}^{2-2 \alpha_{2}} e^{\eta_{b 2}},
$$

where the index 1 or 2 refers to the outermost flux surface of each individual loop. Moreover, when matching the twoloop system, one also needs to ensure that the loops are matched to the background plasma of the same scale height. The scale-height condition demands that

$$
\psi_{1}^{\alpha_{1}}=\psi_{2}^{\alpha_{2}} .
$$

Equations (22) and (23) can be solved for the two unknowns $\psi_{1}$ and $\psi_{2}$. That is, with given sets of parameters $\left(c, c_{1}, a\right)$, it is only some particular flux surfaces that can be matched to the ambient plasma when more than one loop is present in the solar atmosphere.

The characteristic features of the prominence as seen above the solar limb during the solar eclipse are a helmetlike structure lying on the top of the bright prominence and a dark cavity immediately surrounding the bright prominence (Saito \& Tandberg-Hanssen 1973). The helmet-like structure is caused by an outgoing flow that breaks open the magnetic loop/arcade, and cannot be described by our solutions, which address only the static MHD atmosphere; but the cavity associated with the prominence filament loop is clearly illustrated in Figure $3 b$. Taking the temperature of

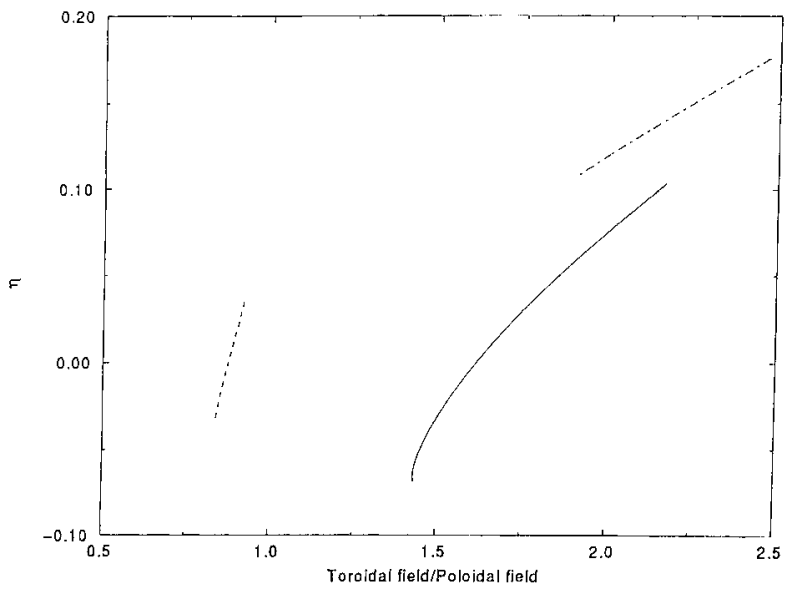

Fig. 4.- Ratios $\left|b_{p}(\eta) / b\right|$ along the reference field lines for the large (solid line) and small (dashed line) loops of Fig. 3, and the arch (dot-dashed line) shown in Fig. 5. the lower corona to be about $10^{6} \mathrm{~K}$ and the local background plasma density at the prominence height $\left(\leq 2 \times 10^{4}\right.$ $\mathrm{km}$ ) to be about $10^{9} \mathrm{~cm}^{-3}$, the internal energy density is about $0.2 \mathrm{ergs} \mathrm{cm}^{-3}$. If we further take the background field to be one-half of the prominence field, whose average value has been measured by the Hanle effect (Leroy 1989) to be about $6 \mathrm{G}$ in the quiescent region, the background magnetic pressure will turn out to be about 0.35 ergs $\mathrm{cm}^{-3}$, only somewhat larger than the equipartition value. Hence, we let the background plasma $\beta=1$ for evaluating the plasma density displayed in Figure $3 b$. Of course, with a lower plasma $\beta$ in the background corona, the plasma density will be correspondingly lower. At any rate, the background open atmosphere should have contained plasmas in motion, a condition that cannot be treated within the present framework; therefore, one needs to interpret the present result for the open atmosphere with caution.

Finally, we should mention that the cores of the two self-similar loops are singular. To avoid unphysical singularities, we have removed the magnetic fields in the cores and filled the space with cool unmagnetized plasmas. These cores may be the gas channels that serve to house the cool gases siphoned from the lower atmosphere; this issue will be discussed further in $\S 4$.

\subsection{Complex-Prominence Solutions}

High-resolution observations show that there are fine structures above the coronal cavities (Saito \& TandbergHanssen 1973). The primary features of the fine structures appear to be arches above the coronal cavity. These arches contain gases of different densities, which appear above the limb as discrete bands in the line-emission intensity. In this section, we attempt to insert a magnetic structure from a different family of self-similar solutions above the smaller prominence loop of Figure 3 in order to represent an overlying magnetic arch, which appears as a dark band. We let the top of the outermost field line of the prominence loop touch on the top of the innermost field line of the overlying arch. This mimics the squeezing of field lines onto a preexisting magnetic arch by the prominence loop when it rises from beneath the photosphere. This additional condition requires that

$$
\left(\eta_{t 1}-\eta_{b 1}\right) \psi_{1}^{\alpha_{1}}=\left(\eta_{t 2}-\eta_{b 2}\right) \psi_{2}^{\alpha_{2}}
$$

where the indices 1 and 2 refer to the two barely touching field lines in question.

Since the overlying arch has a low plasma pressure, it is natural to choose its $c$ to be as small as possible. In addition, since the magnetic fields are relatively strong, the magnetic arch must be relatively rigid, with a relatively small field-line curvature. We therefore let the top part of the loop represent the arch and the bottom part be buried underneath the photosphere. Integration of equation (13) shows that smaller values of $c_{1}$ also give rise to geometrically larger loops, consistent with what has been shown in Figure 3. We thus again choose $c_{1}=0.33$. With a smaller value of $c$ than used for the larger loop in Figure 3, the density of the arcade can quickly decrease to become negative on the sides of the loop. Nonetheless, the amplitude $a$ can be adjusted in order to control the rate at which the density decreases with depth. In Figure 5, we choose $a=1.47$, which gives a rate sufficiently slow that the density, although low, still remains positive at the feet of the arch. 
(a)

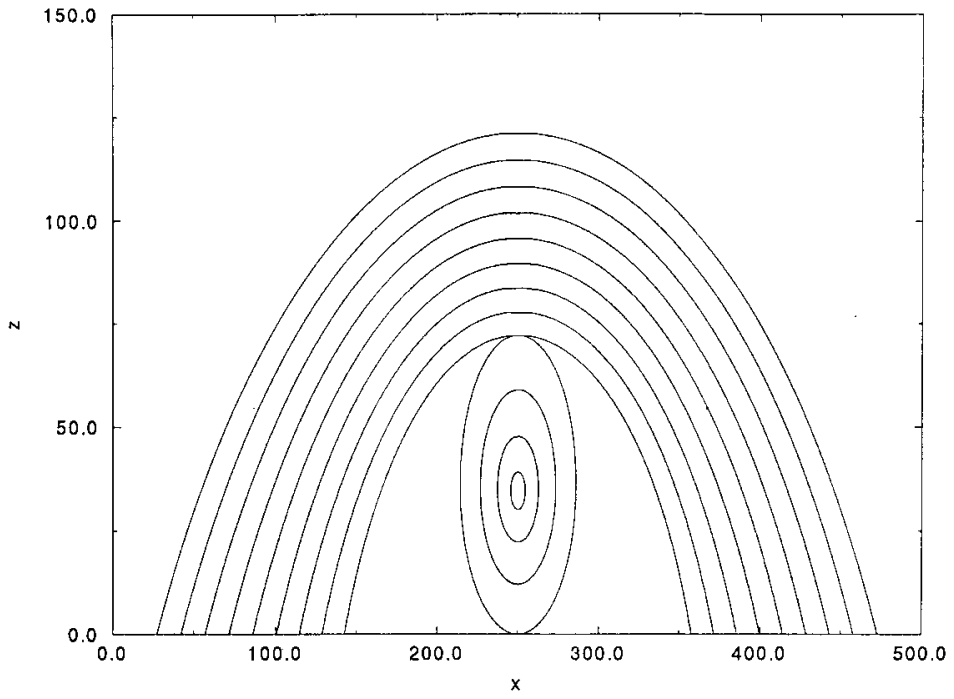

(b) $\log ($ Density)

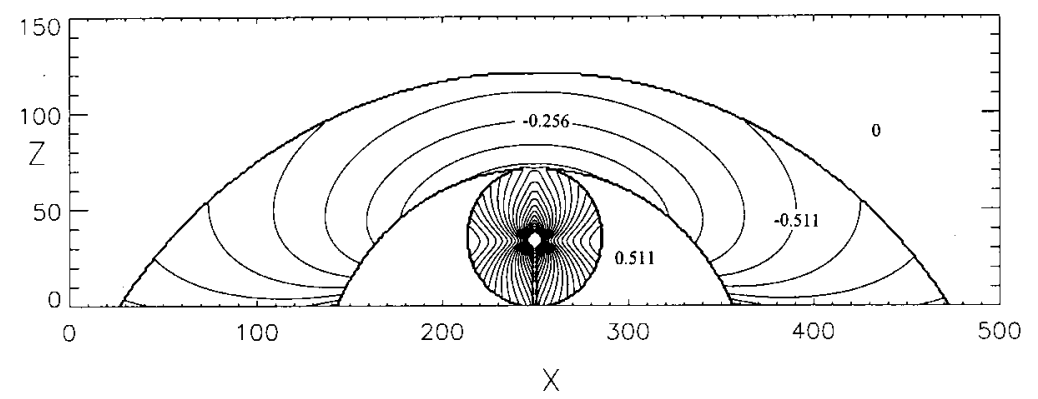

(c) $\log ($ Temperature)

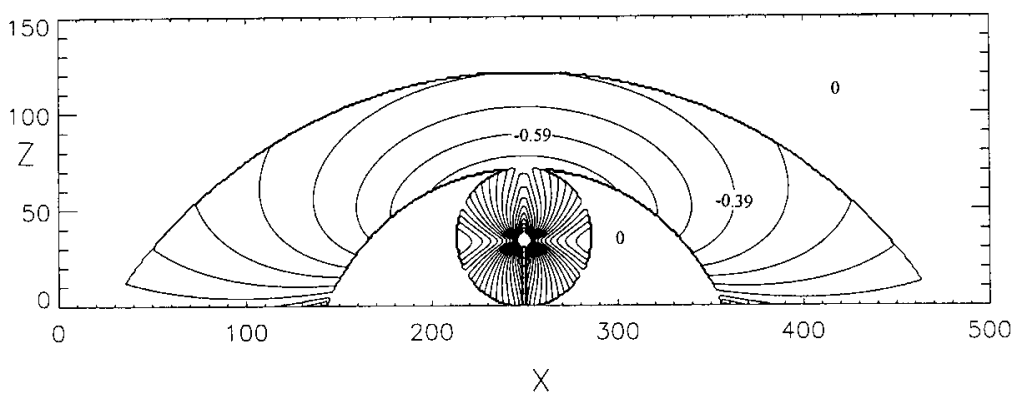

FIG. 5.-Equilibrium configuration of an overlying magnetic arcade in contact with a newly emerged prominence loop. Panels are as in Fig. 3. The adjacent $\log _{10}(\rho)$ contours differ by 0.13 , and those for $\log _{10}(T)$ differ by 0.1 .

To match the two solutions, we need to know not only what $\psi_{1}$ and $\psi_{2}$ ought to be, but also at what level the feet of the arcade, $\eta_{b 1}$, are located. This can be obtained from equation (24), together with equations (22) and (23). Differing from the case discussed in $\S 3.1$, we may have two different background temperatures, one in the open region and the other in the closed region in between the two magnetic structures. In Figure 5, we let the two temperatures be the same, and we also let the feet of the arch assume the background temperature $\left(\sim 10^{6} \mathrm{~K}\right)$. This choice can yield a relatively cool overlying arch of relatively low density. Finally, the outermost field line of the overlying arch can be located anywhere. Once its value of $\psi$ is chosen, the temperature of the background solar atmosphere is then fixed. The dot- dashed line in Figure 4 shows the ratio $\left|b_{p}(\eta) / b\right|$ of the overlying arcade field. Figure 5 shows various aspects of such a composite-prominence complex.

\section{DISCUSSIONS AND CONCLUSIONS}

From the results of this analysis, we find that the highdensity, low-temperature gas sheet suspended at the bottom of the closed magnetic loop pertains to an asymptotically weak magnetic cusp arising from the singularity of equation (13) at $\xi=0$. Such a configuration has traditionally been termed the inverse polarity (Kuperus \& Raadu 1974). The weak cusp is also consistent with the magnetic field observations, in which the prominence field is almost horizontal and the angle discontinuity across the gas sheet is small 
(Bommier, Leroy, \& Sahal-Brechot 1986). For valid selfsimilar solutions, we find that the loop heights are always relatively small, less than $30 \%$ of the (gas + magnetic) pressure scale height of the fiducial gas, $L_{0}$. When the fiducial gas is taken to be the background magnetized coronal plasma, $L_{0}$ can be as large as $10^{5} \mathrm{~km}$, and our quiescent prominence solutions always correspond to the low-lying prominences.

Let us begin the discussion of our prominence solutions by first examining their gross features. From the contour plots of Figures $3 c$ and $5 c$, the temperature assumes the highest value on the sides of the prominence loop and the lowest value at the prominence sheet. From these results, their temperature ratio is generally about several hundred. Since the temperatures of the hot spots are 3-8 times higher than that of the background coronal temperature of about $10^{6} \mathrm{~K}$, the prominence temperature is therefore on the order of $10^{4} \mathrm{~K}$. Figures $3 b$ and $5 b$ also show that the density is highest at the core of the prominence loop and lowest on the loop sides. Their ratio also amounts to several hundred. Since the lowest density spots have a density about 10 times lower than that of the background coronal plasma, the loop core is therefore several tens denser than the background. If one takes the local density of the background lower corona to be $10^{9} \mathrm{~cm}^{-3}$, the dense core will have a density of several $\times 10^{10} \mathrm{~cm}^{-3}$, and the low-density hot spots a density of $10^{8} \mathrm{~cm}^{-3}$. A better measure of the prominence density is the column density across the prominence sheet. Taking the width of the prominence sheet to be about $1 / 10$ of the loop width, or $4 \times 10^{3} \mathrm{~km}$, together with the above quoted prominence density, we find the maximum column mass density to be about $5 \times 10^{-5} \mathrm{~g}$. The scaling of the column density along the prominence sheet is proportional to $\rho r$, and according to the scaling relations immediately preceding equation (1), the column density decreases with vertical distance from the loop core as $|z|^{-c_{1}}$, where $c_{1} \equiv$ $2(1-1 / \alpha)$. These predictions are not in disagreement with the general results obtained by observations.

We proceed to discuss the details of our solutions. The physics behind a prominence configuration can generally be pictured as follows. Under the force of gravity, the light magnetized gas is subject to a buoyancy force, which lifts up the overall magnetic loops. Although the plasma is trapped within each individual loop, it can nevertheless move along the field lines and gravitationally condense at the bottom of each loop, thereby helping to yield a coronal cavity, and the concentration of gases at the bottom of a magnetic loop will inevitably lead to an upward-curved magnetic cusp, whose tension force balances the gravity. However, observations show that the total mass of the gas condensed within the prominence sheet is substantially greater than that of the gas originally occupying the coronal cavity (TandbergHanssen 1974). Hence, additional gas must be supplied from the lower atmosphere by some means. Although early limb observations indicated that the overall flows in prominences are directed downward (Engvold 1976), more recent $\mathrm{H} \alpha$ and $\mathrm{He}$ I observations of the solar disk show that upward and horizontal flows also occur (Schmieder et al. 1984; Simon et al. 1986; Engvold \& Keil 1986), and they may be related to the siphoned gas. The siphon mechanism has been suggested to be operative in extracting additional photospheric gases into the prominence (Saito \& Hyder 1968; Saito \& Tandberg-Hanssen 1973; Lites et al. 1995). If this is indeed so, the suction force for siphoning is likely to originate from a location at which the magnetic field is locally weak and the magnetic pressure low. To find such a site, we have suggested that one choose the central core immediately above the prominence sheet to be cut off from the singular self-similar solution and replaced by a field-free gas, as shown in Figures 3 and 5. The surrounding strong magnetic fields can physically serve to thermally insulate the dense cool gas from the surrounding hot plasmas.

This existence of a high-density gas tube at the top of the gas sheet is further supported by the density measurements. From the $\mathrm{H} \alpha$ observations on the solar disk, the projection of solar prominences often shows that the darkest region, or the highest density light-absorbing region, appears to be located at the top of the prominence sheet. The gas density sharply decreases immediately above this darkest strip, but decreases slowly beneath it (see, e.g., Malherbe 1989). Such a tendency is consistent with our gray-scale density plots given in Figure 6. Related magnetic-field measurements show that the field strength at the prominence sheet increases with height (Leroy, Bommier, \& Sahal-Brechot 1983). They further support the picture of our prominence solutions in which the field strength increases toward the core of the prominence loop from below.

The eclipse white-light and emission-line observations above the solar limb also show the association of the highdensity prominence sheet with coronal fine structures, which include, notably, a low-density cavity and discrete bands of brightness contrast (Saito \& Tandberg-Hanssen 1973). These features can also be found in our model solution shown in Figure $6 b$. The discrete dark bands are probably associated with the overlying magnetic arcades, the gases of which have been squeezed out when a new prominence loop emerges from the subphotospheric level. The bright bands may be the regions of enhanced plasma density immediately above the highest density gas core. These correspond to the broadly distributed regions of density enhancement surrounding the prominence sheets (see Fig. 6), which has a lower temperature than the background coronal plasma (see Figs. $3 c$ and $5 c$ ). These regions may efficiently absorb thermal radio emission from the lower corona, thereby yielding a broader radio dark band cospatial with the narrower $\mathrm{H} \alpha$ dark filament when viewed on the disk. Both centimeter and millimeter radio observations indeed show such a feature of radio dark bands, and these radio dark bands are usually several times larger than the $\mathrm{H} \alpha$ dark filament in the projected area (Hiei et al. 1986; Kundu 1986).

Our solution also gives another interesting feature, in that the plasma temperature becomes high on the sides of the prominence loop (see Figs. $3 c$ and $5 c$ ). In our model, this feature arises as a consequence of diminishing plasma density at finite plasma pressure in those locations. The fact that the plasma density is locally low on the sides of the magnetic loop is the result of the vanishingly small vertical magnetic force. For the plasma to be supported against gravity, it locally relies solely on the gas pressure, which has a large scale height $\left(L_{0} \sim 10^{5} \mathrm{~km}\right)$; hence, only a small amount of gas is allowed to be present. In addition, the loop plasma has a plasma $\beta$ on the order of unity, and to be pressure balanced with the surrounding plasma, it is necessary that the pressure be rather uniform along the field lines, yielding a locally high temperature. This hot feature should therefore be generic for any MHD equilibrium of the lowlying solar prominences. In fact, the VLA radio continuum 
(a) $\log$ (Density)

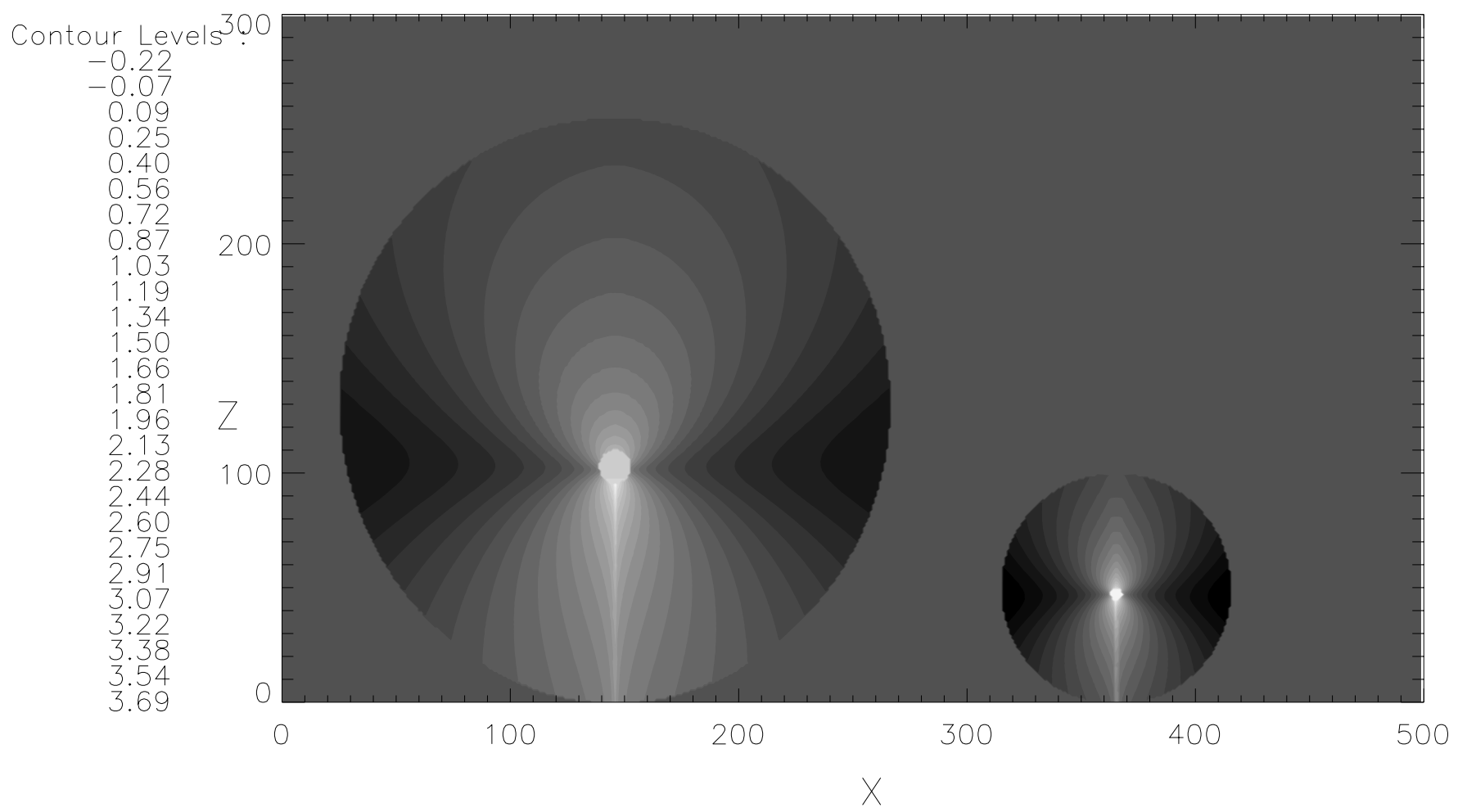

FIG. $6 a$

(b) $\log ($ Density)

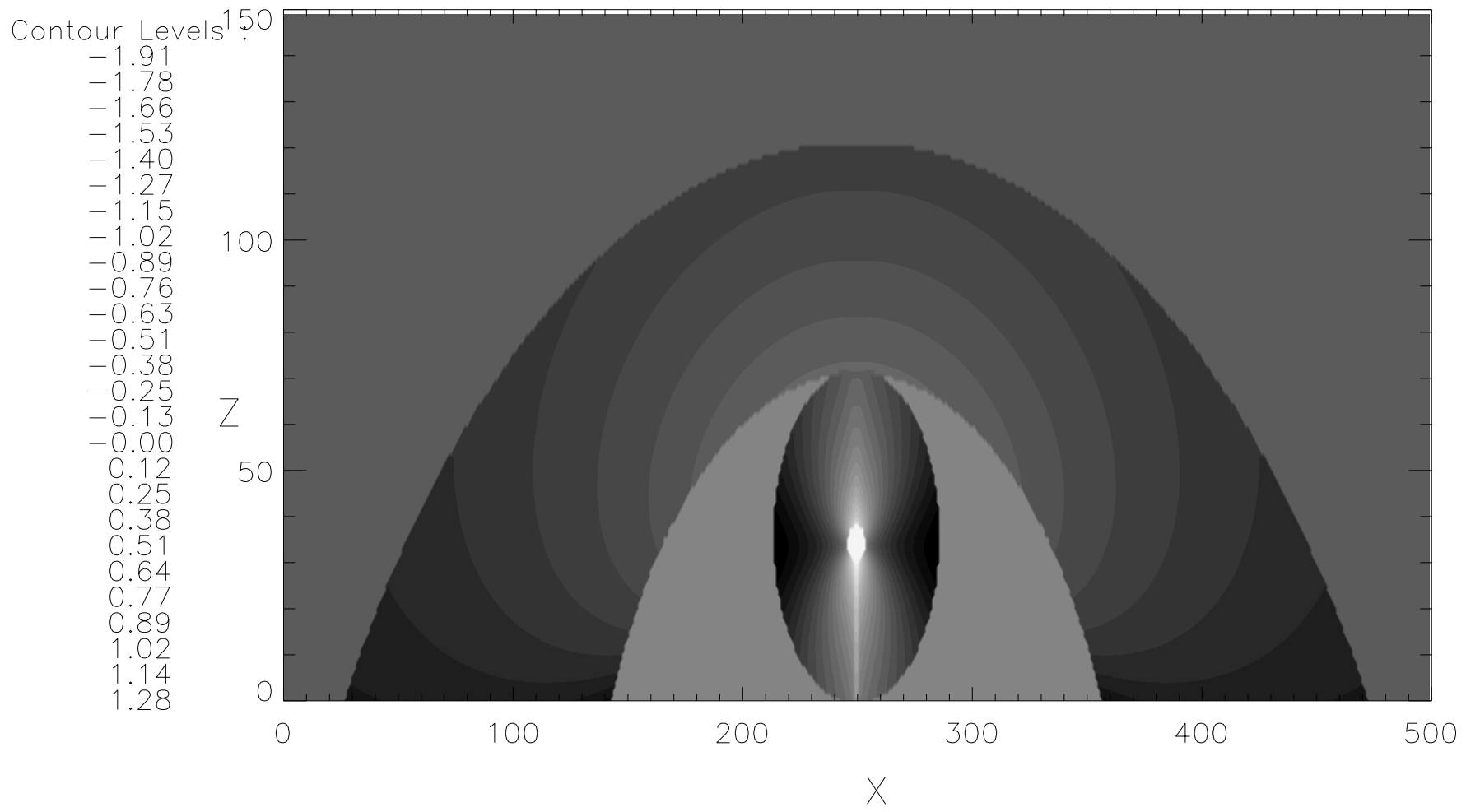

FIG. $6 b$

FIG. 6.-Gray-scale plots of $\log _{10}(\rho)$ for $(a)$ the multisimple loops and $(b)$ the complex loops shown in Figs. 3 and 5, respectively 
(15 GHz) observations show that two bright ribbons of width about $7000 \mathrm{~km}$ are located on both sides of the prominence sheet (Gary 1986). The radio continuum may be emitted from the hot spots located on the two sides of the prominence loop. (Note that the radio continuum arises from the Rayleigh-Jeans tail of the thermal emission, whose intensity is proportional to the temperature and independent of the plasma density.) Since our prominence loop generally has a height of about a few $\times 10^{4} \mathrm{~km}$, the fact that the bright ribbons have a width of about $7000 \mathrm{~km}$ does seem to be a reasonable value when our solutions for the hot spots are compared (see Figs. $3 c$ and $5 c$ ). Note that the energy balance has not been considered in the present analysis. What if the plasma cannot be heated to a temperature as high as $10^{7} \mathrm{~K}$ ? If this is indeed the case, the only logical possibility is that the plasma $\beta$ in the magnetic loop would be much smaller than unity, so that the gas pressure is dropped from the consideration of force balance. If this occurs, whether there can ever exist a prominence in such a low- $\beta$ loop would be highly questionable. At any rate, these hot spots have a sufficiently high temperature to be the soft $\mathrm{X}$-ray source in the quiet Sun. High-resolution X-ray imaging should be able to test this prediction.

In sum, the present analytical model does provide a fair picture for the prominence equilibria based on the balance of magnetic, gravitational and pressure forces. Although the thermodynamic state is not considered, the solutions obtained in this work nonetheless are in several respects in qualitative agreement with the observations, and they may serve as a good starting point in understanding the physics of quiescent prominences. In particular, because of the simplicity of the governing equation (eq. [13]), these analytical solutions can be obtained with sufficient precision for the numerical simulation to set up an accurate initial equilibrium for investigating its MHD stability and long-term evolution.

This work is supported in part by the National Science Council of Taiwan, under grants NSC 87-2112-M008-010 and NSC 88-2112-M008-039.
Blandford, R. D., \& Payne, D. G. 1982, MNRAS, 199, 88

Bommier, V., Leroy, J. L., \& Sahal-Brechot, S. 1986, A\&A, 156, 79

Cattaneo, F., Chiueh, T., \& Hughes, D. W. 1990a, MNRAS, 247, 6p 1990b, J. Fluid Mech., 219,

Chiueh, T. 1998, in preparation

Chiueh, T., \& Zweibel, E. G. 1987, ApJ, 317, 900 1989, ApJ, 338, 1158

Engvold, O. 1976, Sol. Phys., 49, 283

Engvold, O., \& Keil, S. 1986, in Coronal and Prominence Plasmas, ed. A. I. Poland (NASA Conf. Publ. 2442) (Washington: NASA), 169

Field, G. B. 1965, ApJ, 142, 531

Gary, D. 1986, in Coronal and Prominence Plasmas, ed. A. I. Poland (NASA Conf. Publ. 2442) (Washington: NASA), 121

Gibson, S. E., \& Low, B. C. 1998, ApJ, 493, 460

Hiei, E., Ishiguro, M., Kosugi, T., \& Shibasaki, K. 1986, in Coronal and Prominence Plasmas, ed. A. I. Poland (NASA Conf. Publ. 2442) (Washington: NASA), 109

Hildner, E. 1974, Sol. Phys., 35, 123

Kundu, M. R. 1986, in Coronal and Prominence Plasmas, ed. A. I. Poland (NASA Conf. Publ. 2442) (Washington: NASA), 117

Kuperus, M., \& Raadu, M. 1974, ApJ, 31, 189

Leroy, J. L. 1989, in Dynamics and Structures of Quienscent Prominences, ed. E. R. Priest (Dordrecht: Reidel), 77

\section{REFERENCES}

Leroy, J. L., Bommier, V., \& Sahal-Brechot, S. 1983, Sol. Phys., 83, 135

Li, Z. Y., Chiueh, T., \& Begelman, M. C. 1992, ApJ, 394, 459

Lites, B. W., Low B. C., Martinez-Pillet, V., Seagraves, P., Skumanich, A., Frank, Z. A., Shine, R. A., \& Tsuneta, S. 1995, ApJ, 446, 877

Low, B. C. 1996, Sol. Phys., 167, 217

Malherbe, J. M. 1989, in Dynamics and Structures of Quienscent Prominences, ed. E. R. Priest (Dordrecht: Reidel), 115

Martin, S. F. 1989, in Dynamics of Quiescent Prominences, ed. V. Ruzdjak \& E. Tabdberg-Hanssen (Berlin: Springer), 1

Saito, K., \& Hyder, C. 1968, Sol. Phys., 5, 61

Saito, K., \& Tandberg-Hanssen, E. 1973, Sol. Phys., 31, 105

Schmieder, B., Malherbe, J. M., Mein, P., \& Tandberg-Hanssen, E. 1984, A\&A, 136, 81

Sedov, L. I. 1959, Similarity and Dimensional Methods in Mechanics (New York: Academic)

Simon, G., Schmieder, B., Demoulin, P., \& Poland, A. I. 1986, A\&A, 166, 319

Tandberg-Hanssen, E. 1974, Solar Prominences (Dordrecht: Reidel) 1995, The Nature of Solar Prominences (Dordrecht: Kluwer)

Tang, F. 1987, Sol. Phys., 107, 223

Zirin, H. 1988, Astrophysics of the Sun (Cambridge: Cambridge Univ. Press) 